\title{
A phase 2 double-blind placebo-controlled 24-week treatment clinical study of the p38 alpha kinase inhibitor neflamapimod in mild Alzheimer's disease
}

Niels D. Prins ${ }^{1,2^{*}}$ D, John E. Harrison ${ }^{1,3,4}$, Hui-May Chu ${ }^{5}$, Kelly Blackburn ${ }^{6}$, John J. Alam ${ }^{6}$, Philip Scheltens ${ }^{1,2}$ and for the REVERSE-SD Study Investigators

\begin{abstract}
Background: In preclinical studies, p38a kinase is implicated in Alzheimer's disease (AD) pathogenesis. In animal models, it mediates impaired synaptic dysfunction in the hippocampus, causing memory deficits, and is involved in amyloid-beta (Aß) production and tau pathology.

Methods: The REVERSE-SD (synaptic dysfunction) study was a multi-center phase 2, randomized, double-blind, placebo-controlled trial of the p38a kinase inhibitor neflamapimod; conducted December 29, 2017, to June 17, 2019; 464 participants screened, and 161 randomized to either 40 mg neflamapimod (78 study participants) or matching placebo (83 study participants), orally twice daily for 24 weeks. Study participants are as follows: CSF ADbiomarker confirmed, Clinical Dementia Rating (CDR)-global score 0.5 or 1.0, CDR-memory score $\geq 0.5$, and MiniMental State Examination (MMSE) 20-28. The primary endpoint was the improvement in episodic memory, assessed by combined change in Z-scores of Hopkins Verbal Learning Test-Revised (HVLT-R) Total and Delayed Recall. Secondary endpoints included change in Wechsler Memory Scale-IV (WMS) Immediate and Delayed Recall composites, CDR-SB, MMSE, and CSF biomarkers [total and phosphorylated tau (T-tau and p-tau 181 ), $A \beta_{1-40}, A \beta_{1-42}$, neurogranin, and neurofilament light chain].
\end{abstract}

Results: At randomization, the mean age is $72,50 \%$ female, $77 \%$ with CDR-global score 0.5 , and mean MMSE score 23.8. The incidence of discontinuation for adverse events and serious adverse events (all considered unrelated) was $3 \%$ each. No significant differences between treatment groups were observed in the primary or secondary clinical endpoints. Significantly reduced CSF levels with neflamapimod treatment, relative to placebo, were evident for Ttau [difference $(95 \% \mathrm{Cl}):-18.8(-35.8,-1.8) ; P=0.031]$ and $p-\operatorname{tau}_{181}[-2.0(-3.6,-0.5) ; P=0.012]$, with a trend for neurogranin $[-21.0(-43.6,1.6) ; P=0.068]$. In pre-specified pharmacokinetic-pharmacodynamic (PK-PD) analyses, subjects in the highest quartile of trough plasma neflamapimod levels demonstrated positive trends, compared with placebo, in HLVT-R and WMS.

(Continued on next page)

\footnotetext{
* Correspondence: n.prins@brainresearchcenter.nl

'Alzheimer Center, Department of Neurology, Amsterdam UMC, Amsterdam,

The Netherlands

${ }^{2}$ Brain Research Center, Amsterdam, The Netherlands

Full list of author information is available at the end of the article
}

\section{$\triangle B M C$}

(c) The Author(s). 2021 Open Access This article is licensed under a Creative Commons Attribution 4.0 International License, which permits use, sharing, adaptation, distribution and reproduction in any medium or format, as long as you give appropriate credit to the original author(s) and the source, provide a link to the Creative Commons licence, and indicate if changes were made. The images or other third party material in this article are included in the article's Creative Commons licence, unless indicated otherwise in a credit line to the material. If material is not included in the article's Creative Commons licence and your intended use is not permitted by statutory regulation or exceeds the permitted use, you will need to obtain permission directly from the copyright holder. To view a copy of this licence, visit http://creativecommons.org/licenses/by/4.0/ The Creative Commons Public Domain Dedication waiver (http://creativecommons.org/publicdomain/zero/1.0/) applies to the data made available in this article, unless otherwise stated in a credit line to the data. 
(Continued from previous page)

Conclusions and relevance: A 24-week treatment with 40 mg neflamapimod twice daily did not improve episodic memory in patients with mild AD. However, neflamapimod treatment lowered CSF biomarkers of synaptic

dysfunction. Combined with PK-PD findings, the results indicate that a longer duration study of neflamapimod at a higher dose level to assess effects on AD progression is warranted.

Trial registration: ClinicalTrials.gov identifier: NCT03402659. Registered on January 18, 2018

Keywords: p38 MAPK, Alzheimer's, Episodic memory, Clinical trial, CSF biomarkers, Synaptic dysfunction

\section{Background}

Synaptic dysfunction has emerged as a therapeutic target for Alzheimer's disease (AD), particularly in the early stages of disease [1-3]. In animal models of neurodegenerative disease intervention, approaches that target stress-activated intracellular pathways have shown the ability to both restore function and prevent further neuronal loss, if initiated early in the disease process, after neuronal loss has begun but before it has become widespread [4]. The alpha isoform of p38 mitogen-activatedprotein kinase $(\mathrm{p} 38 \alpha)$ is an intracellular protein that is considered to be a leading therapeutic target for treating synaptic dysfunction in AD [5-9]. Expression of p38 $\alpha$ in the neuron is associated with the formation of pathological amyloid-beta $(A \beta)$, inflammation, and tauinduced synaptic dysfunction [7, 10-17]. Studies in several distinct animal models, each driven either by $A \beta$, inflammation, or tau, showed that spatial learning deficits are reversed with small molecule inhibitors of $p 38 \alpha$ kinase activity [18-20], providing direct evidence that p38 $\alpha$ inhibition could be beneficial in AD. Moreover, genetic reduction of neuronal $\mathrm{p} 38 \alpha$ levels in amyloidprecursor-protein overexpressing transgenic mice improved synaptic transmission, decreased memory loss, and reduced amyloid pathology [21, 22]. Genetically, suppressing p38 $\alpha$ expression in mice also prevented agerelated decline in hippocampal function [23, 24]. This is consistent with a human genetic linkage study identifying the p38 $\alpha$ pathway as a modifier of age-related decline in episodic memory [25] and mechanistic studies showing that $\mathrm{p} 38 \alpha$ modulates memory formation [26].

The investigational drug neflamapimod is an oral, brain-penetrant, selective $\mathrm{p} 38 \alpha$ inhibitor that reversed functional deficits in performance in the Morris Water Maze in aged rats [18]. Two small (25 patients in total) phase 2a clinical studies of neflamapimod in early $\mathrm{AD}$, without a placebo control, showed good tolerability, adequate drug concentrations in CSF, and trends for improving episodic memory [27, 28]. To further evaluate its effects on synaptic dysfunction, as evaluated by tests of episodic memory and CSF biomarkers, we performed a placebo-controlled clinical trial, named REVERSE-SD (synaptic dysfunction), of this p38 $\alpha$ kinase inhibitor in patients with mild $\mathrm{AD}$. The primary objective of the trial was to determine whether 24 weeks of treatment with neflamapimod improves episodic memory (a direct measure of synaptic dysfunction in the hippocampus) and decreases levels of CSF biomarkers considered to be associated with synaptic dysfunction in AD.

\section{Methods}

\section{Participants and study design}

This was a multi-center phase 2, randomized, parallelgroup, double-blind placebo-controlled trial conducted in 38 centers in the US (16 centers, $45 \%$ of participants), the UK (11, 32.5\%), the Netherlands (3, 12.5\%), Czech Republic (5, 6\%), and Denmark (3, 4\%). One hundred sixty-one patients were randomized, of whom 78 received neflamapimod and 83 placebos. The first participant was enrolled on December 29, 2017, and the last visit occurred on June 17, 2019. The study was conducted in accordance with Good Clinical Practice guidelines [29] and the Declaration of Helsinki [30]. Applicable local/central ethics committee or IRB approvals were obtained, and all participants provided written informed consent.

During the treatment period, participants attended study center visits on week 3 , week 6 , week 12 , week 18 , and week 24. Participants took study drug twice daily with a meal for 24 weeks. The main clinical outcome measures, the Hopkins Verbal Learning Test-Revised (HVLT-R) and the Wechsler Memory Scale-IV (WMS), were conducted on day 1 (baseline) and on weeks 6,12 , and 24. Lumbar puncture to collect CSF was conducted during the screening period and repeated at week 24 .

Participants eligible for randomization were 55 to 85 years of age; had a Clinical Dementia Rating Scale (CDR) Global Score of 0.5 or 1.0, a CDR memory subscore $\geqq 0.5$, CSF $\mathrm{A} \beta_{42}<1000 \mathrm{pg} / \mathrm{mL}$, and CSF $\mathrm{p}-\mathrm{tau}_{181} /$ $\mathrm{A} \beta_{42}$ ratio $>0.024$ by Roche Elecsys ${ }^{\circ}$ assay; and MiniMental State Examination (MMSE) score of 20 to 28, inclusive. Participants on a single-drug AD therapy (cholinesterase inhibitors or memantine, dual therapy excluded) could remain on that background AD therapy if on a stable dose for $\geq 2$ months and the dose was not changed during the trial. Randomization was conducted by a central Interactive Web Response System and 
stratified by CDR global score (0.5/1.0) and use of background AD therapy (yes/no).

All participants received their first dose of the study drug at the clinical site. Thereafter, participants were instructed to take a study drug were taken within 30 min following a meal or snack (i.e., breakfast and dinner) no less than $8 \mathrm{~h}$ apart and at approximately the same times each day throughout the study. The count of returned capsules at each visit was reviewed, and any apparent discrepancies between the quantity of capsules returned and the number expected based on the dosing schedule were discussed with the subject to ensure an understanding of dosing instructions. Participants were also provided with identification cards on which they recorded the date and time of study drug administration prior to each scheduled study center visit.

\section{Primary outcome measure}

The HVLT-R [31] is a word list verbal learning test that evaluates episodic learning and memory. Each of the six alternative forms of the test consists of a 12-item word list, composed of four words from each of the three semantic categories. Total Recall score (scored 0-36, the sum of three immediate recall trials) and Delayed Recall score (scored 0-12; single recall, 20 to $25 \mathrm{~min}$ after initial trials) were assessed. To minimize learning effects, form A was utilized on day 1 (baseline), form B on week 6 , form $C$ on week 12 , and form D on week 24 . We calculated $Z$-scores for Total Recall and Delayed Recall scores on the HVLT-R.

\section{Secondary outcome measures \\ Cognitive measures}

Participants were also assessed using episodic memory components of the WMS [32]. Performance was assessed using composite measures of both immediate and delayed recall. The WMS Immediate Recall composite score at each testing session consisted of the sum of the scores for Logical Memory (LM) I, Verbal Paired Associates (VPA) I, and Visual Reproduction (VR) I. The WMS Delayed Recall composite score consisted of the sum of LM II, VPA II, and VR II. To obtain data for designing future studies of longer duration, CDR sum-ofboxes (CDR-SB) and MMSE scores were also assessed.

\section{Cerebrospinal fluid measures}

Kits for CSF sample collection were provided to the sites and aliquots for endpoint biomarker analyses were stored at $-80^{\circ} \mathrm{C}$ until the end of the study. CSF protein levels of total tau (T-tau), phosphorylated tau ( $\mathrm{p}$-tau tri1 $_{1}$ ), $A \beta_{40}, A \beta_{42}$, neurogranin, and neurofilament light chain (NfL) were determined with commercially available enzyme-linked immunosorbent assay kits in the laboratory of Charlotte Teunissen at Amsterdam UMC,
Amsterdam, NL (Roche Elecsys ${ }^{\circ}: \mathrm{T}$-tau, P-tau $181, A \beta_{42}$; Euroimmun: $A \beta_{40}$; $A D x$ Neuroscience: neurogranin; Uman Diagnostics: NfL).

\section{Pharmacokinetics}

For trough plasma drug concentration determinations, on day 21, participants were instructed and called the day before to not take their morning dose of study drug at home; instead, that dose was administered at the study center and blood samples collected for pharmacokinetic (PK) testing immediately prior to study drug administration. In addition, that the participant had taken their second dose of study drug the evening before was confirmed and the time of that dose was recorded. Drug concentrations were determined utilizing a validated LC-MS/MS assay [33].

\section{Statistical analysis}

The efficacy population included the 160 participants who received their first dose of study drug (in all cases at the clinical site) and had at least one post-dose efficacy assessment. The primary efficacy endpoint was the combined change in Z-score of Total Recall and Delayed Recall on the HVLT-R in neflamapimod-treated subjects, compared with placebo recipients, at week 24 . We analyzed the relationship between neflamapimod use and change in the primary endpoint, as well as in WMS and CDR-SB scores, using Mixed Model for Repeated Measures (MMRM) with fixed effects for treatment, background AD therapy, CDR-Global Score of 0.5 versus 1.0, scheduled visit (nominal) and scheduled visit by treatment interaction, random effect for subject and baseline $Z$-score as a covariate. Least-square means (LSM) and 2-sided 95\% confidence intervals (CI) are provided for treatment group differences and estimated endpoint values by visit. The original sample size of 76 patients per arm provided $90 \%$ power to detect an effect size (ES) of 0.53 and $80 \%$ power to detect an ES of 0.46 on the primary endpoint.

Changes in CSF biomarkers and MMSE scores were compared using an ANCOVA with treatment group, background AD therapy, and baseline CDR-Global Score as main effects and the baseline assessment as the covariate. The results of the ANCOVA are summarized using the treatment group LSMs, the difference between the treatment group LSMs, the 95\% confidence interval for the treatment group difference, and the $p$ value.

\section{Results}

Patients, demographics, and enrollment

Four hundred sixty-four patients entered screening and 161 were randomized; 83 to the placebo group and 78 to the neflamapimod $40 \mathrm{mg}$ group (Fig. 1). Among randomized 


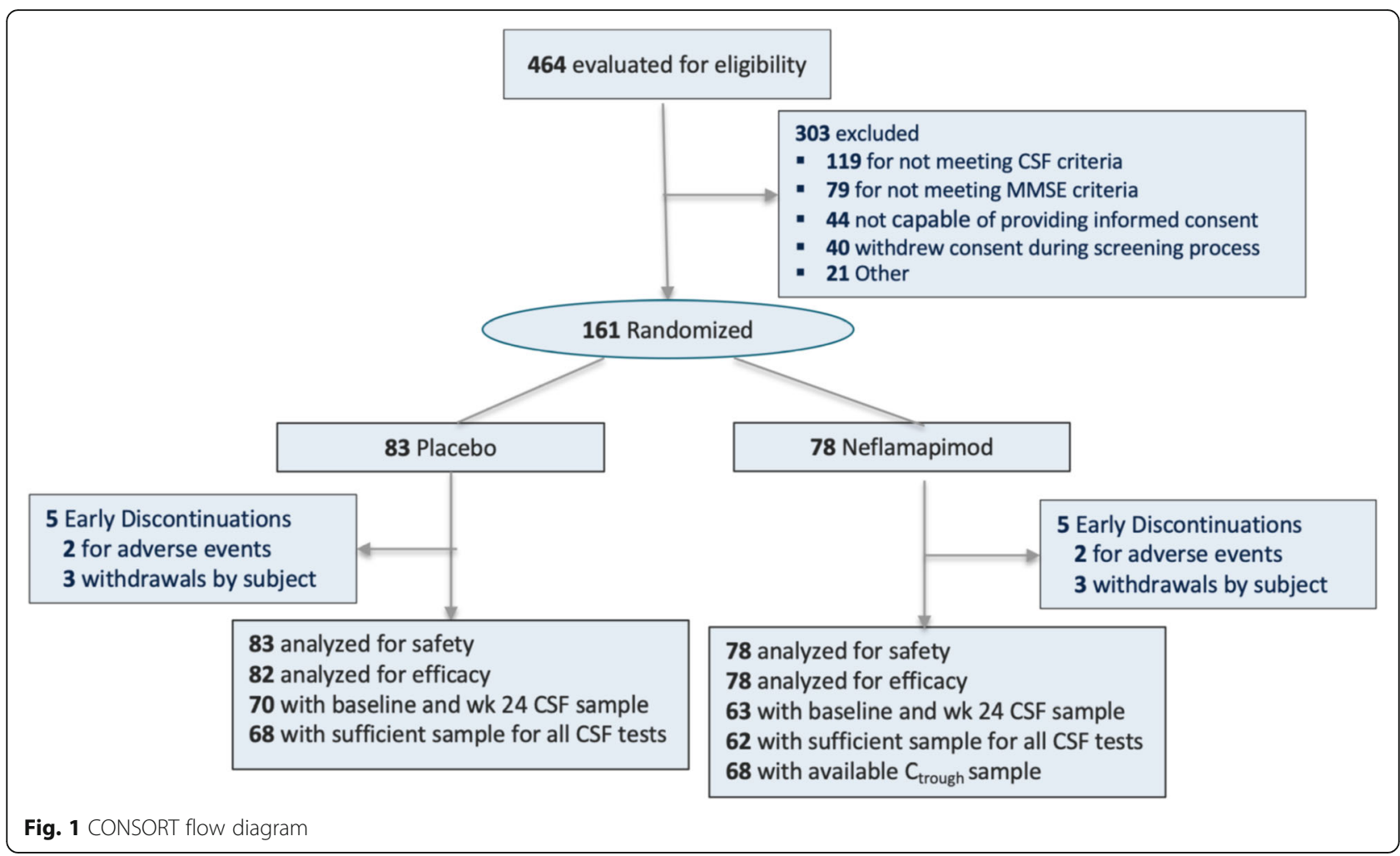

patients, $50 \%$ were males and $50 \%$ females. The mean (SD) age of subjects was 72 (6.8) years. Most subjects were white (156 subjects, 97\%). One hundred twentyfive $(78 \%)$ patients had a global CDR-Global score of 0.5 (mild cognitive impairment) and $36(22 \%)$ had a score of 1 (mild dementia). The mean MMSE score was 23.8 (2.48). All patients randomized received at least one dose of study drug and were included in assessments of safety. All but one placebo recipient had at least one on-treatment efficacy assessment, and so 82 placebo recipients and 78 neflamapimod recipients were included in the efficacy analysis population. Based on counts of returned capsules, $91.8 \%$ of placebo-recipients and $93.6 \%$ of neflamapimodrecipients received greater than $90 \%$ of their planned study doses.

Table 1 gives the baseline characteristics by treatment group and whether the patients were receiving background $\mathrm{AD}$ therapy, a stratification variable in the randomization. The patients receiving background $\mathrm{AD}$ therapy had significantly more advanced disease while, within each stratum (with or without background $\mathrm{AD}$ therapy), there were no significant differences between neflamapimod and placebo recipients for any of the clinical and CSF measures at baseline. Of neflamapimod and placebo recipients, $59 \%$ and $61 \%$, respectively, received background AD therapy (85\% taking a cholinesterase inhibitor and 15\% memantine).

\section{Full efficacy population analyses}

Table 2 gives the results of clinical endpoint analyses of the full efficacy population (Table 2). The primary endpoint was not met, with no significant difference between neflamapimod treatment and placebo in the change from baseline to week 24 in the combined Z-score of Total Recall and Delayed Recall in the HVLT-R. Similarly, no significant differences between neflamapimod and placebo were seen over the same time period in secondary clinical efficacy measures, including WMS Immediate and Delayed Recall composite scores, CDR-SB scores, and MMSE scores.

Figure 2 shows CSF biomarker results in the full efficacy population. Improvements, based on decreased levels of the disease biomarkers CSF T-tau [difference (95\% CI): $-18.8(-35.8,-1.8) ; P=0.031]$ and CSF p-tau 181 $[-2.0(-3.6,-0.5) ; P=0.012]$, were seen over the 24-week neflamapimod treatment period, relative to placebo. Furthermore, CSF neurogranin levels showed a trend [ -21.0 $(-43.6,1.6) P=0.068]$ towards improvement with neflamapimod treatment, relative to placebo administration. Of note, the standard deviation relative to mean baseline level was greater for neurogranin than for either $\mathrm{T}$-tau or $\mathrm{p}$-tau, providing a potential explanation for the higher $p$ value despite a similar proportionate difference for the CSF neurogranin comparison between neflamapimod and placebo groups. 
Table 1 Baseline disease characteristics by background AD therapy

\begin{tabular}{llllll}
\hline & Placebo & & & Neflamapimod \\
\cline { 2 - 3 } \cline { 5 - 6 } & AD therapy $(\boldsymbol{n}=\mathbf{5 1})$ & No AD therapy $(\boldsymbol{n}=\mathbf{3 2})$ & & AD therapy $(\boldsymbol{n}=\mathbf{4 6})$ & No AD therapy $(\boldsymbol{n}=\mathbf{3 2})$ \\
\hline Age & $73(7.8)$ & $72.1(5.9)$ & $72.2(6.8)$ & $68.9(5.6)$ \\
Gender (\% female) & 45.1 & 62.5 & 47.8 & 46.9 \\
ApoE4 (\% positive) & 66.7 & 71.9 & 67.4 & 78.1 \\
HVLT-R Total Recall & $13.4(4.5)$ & $18.3(4.9)$ & $14.8(5.1)$ & $18.6(6.9)$ \\
HVLT-R Delayed Recall & $1.8(2.1)$ & $4.0(3.3)$ & $2.2(2.4)$ & $4.7(4.0)$ \\
WMS Immediate \& Delayed Recall & $58.1(21.8)$ & $84.2(34.1)$ & $61.7(25.0)$ & $92.6(42.0)$ \\
CDR sum of boxes & $3.7(1.6)$ & $2.4(1.5)$ & $3.4(1.4)$ & $2.6(1.0)$ \\
MMSE & $22.7(2.4)$ & $25.2(2.7)$ & $22.9(2.5)$ & $25.1(2.3)$ \\
CSF A $\beta_{40}$ & $10583(3340)$ & $10783(3162)$ & $11099(2926)$ & $11259(3465)$ \\
CSF A $\beta_{42}$ & $545(155)$ & $562(194)$ & $556(138)$ & $602(196)$ \\
CSF p-tau 181 & $35.5(14.2)$ & $30.9(11.0)$ & $37.6(15.1)$ & $33.4(14.4)$ \\
CSF total tau & $360(126)$ & $316(97)$ & $378(132)$ & $337(116)$ \\
CSF neurogranin & $477(238)$ & $427(160)$ & $510(224)$ & $469(161)$ \\
CSF NfL & $1456(640)$ & $1230(694)$ & $1586(760)$ & $1257(530)$
\end{tabular}

Mean (SD), except where percentage is shown. CSF levels are shown as $\mathrm{pg} / \mathrm{mL}$

\section{PK-PD analyses}

All participants randomized to neflamapimod had quantifiable plasma drug concentrations at their day 21 predose plasma sample $\left(C_{\text {trough }}\right)$ determination, indicating that participants had received their dose of study drug the evening before. Figure 3a illustrates the relationship between $C_{\text {trough }}$ and the primary endpoint. In the placebo group, subjects on background AD therapy had a median $Z$-score decline of approximately 0.15 over the 24 weeks in the trial, while those not on background AD therapy had a median of no change over the 24 weeks. There was high inter-subject variability (standard deviation for change in $Z$-score of 0.6 for the placebo group as a whole). In the neflamapimod-treated subjects, examination of the outcomes by $\mathrm{C}_{\text {trough }}$ revealed an apparent threshold effect in the change from baseline to week 24 in the primary endpoint. Thus, subjects with $\mathrm{C}_{\text {trough }}$ levels lower than approximately $4 \mathrm{ng} / \mathrm{mL}$ had a distribution similar to that in the placebo group while, in those with $C_{\text {trough }}$ levels greater than $4 \mathrm{ng} / \mathrm{mL}$, the distribution was shifted upwards with fewer subjects showing a decline, i.e., less disease progression. In a prespecified analysis, the primary endpoint outcome in the neflamapimod subjects in the highest quartile of $\mathrm{C}_{\text {trough }}$ (cut-off $=5.4 \mathrm{ng} / \mathrm{mL}$ ) was compared to placebo recipients and the subjects in the lower three quartiles of neflamapimod $C_{\text {trough }}$. The least-square mean (s.e.m.) change from baseline to week 24 derived from MMRM analysis was $+0.10(0.16)$ in the neflamapimod with $\mathrm{C}_{\text {trough }}>75$ th percentile $(\sim 5 \mathrm{ng} / \mathrm{mL})$, compared to -0.13 (0.1) amongst placebo recipients (difference not significant) and $-0.21(0.11)$ in the neflamapimod subjects with $\mathrm{C}_{\text {trough }}<75$ th percentile.

For the HVLT-R, alternative versions were utilized in each session to minimize learning effects. However, this could not be done for the WMS, because there are no alternative versions. As a result, in the placebo group, there were significant learning effects, especially in subjects not on background AD therapy, resulting in significant improvements in WMS Immediate and Delayed Recall scores from baseline to week 24 (Fig. 3b). However, this learning effect was less evident in subjects on background $\mathrm{AD}$ therapy, presumably because they had more advanced disease. With that limitation, similarly to the relationship for the primary endpoint, neflamapimod subjects with higher $C_{\text {trough }}$ levels appear to have less

Table 2 Changes of clinical endpoints from baseline to week 24

\begin{tabular}{lllll}
\hline Endpoint & Placebo $(\boldsymbol{n}=\mathbf{8 2})$ & Neflamapimod $(\boldsymbol{n}=\mathbf{7 8})$ & Difference in change $(\mathbf{9 5} \%$ Cl) & $\boldsymbol{P}$ value \\
\hline HVLT-R combined Z-score Total and Delayed Recall & $-0.13(-0.27,0.01)$ & $-0.17(-0.38,0.05)$ & $-0.03(-0.23,0.16)$ & NS \\
WMS Immediate \& Delayed Recall & $16.6(11.1,22.1)$ & $16.0(10.5,21.5)$ & $-0.6(-6.0,4.8))$ & NS \\
CDR-SB & $1.0(0.5,1.5)$ & $1.1(0.6,1.7)$ & $0.1(-0.4,0.6)$ & NS \\
MMSE & $-0.5(-1.3,0.3)$ & $-0.8(-1.7,0.1)$ & $-0.3(-1.0,0.5)$ & NS
\end{tabular}

Except for MMSE, results shown derived from mixed model for repeated measures (MMRM) analysis of change from baseline to week 24. MMSE results from ANCOVA of change from baseline to week 24 . Least square means ( $95 \%$ confidence interval) from models are shown 


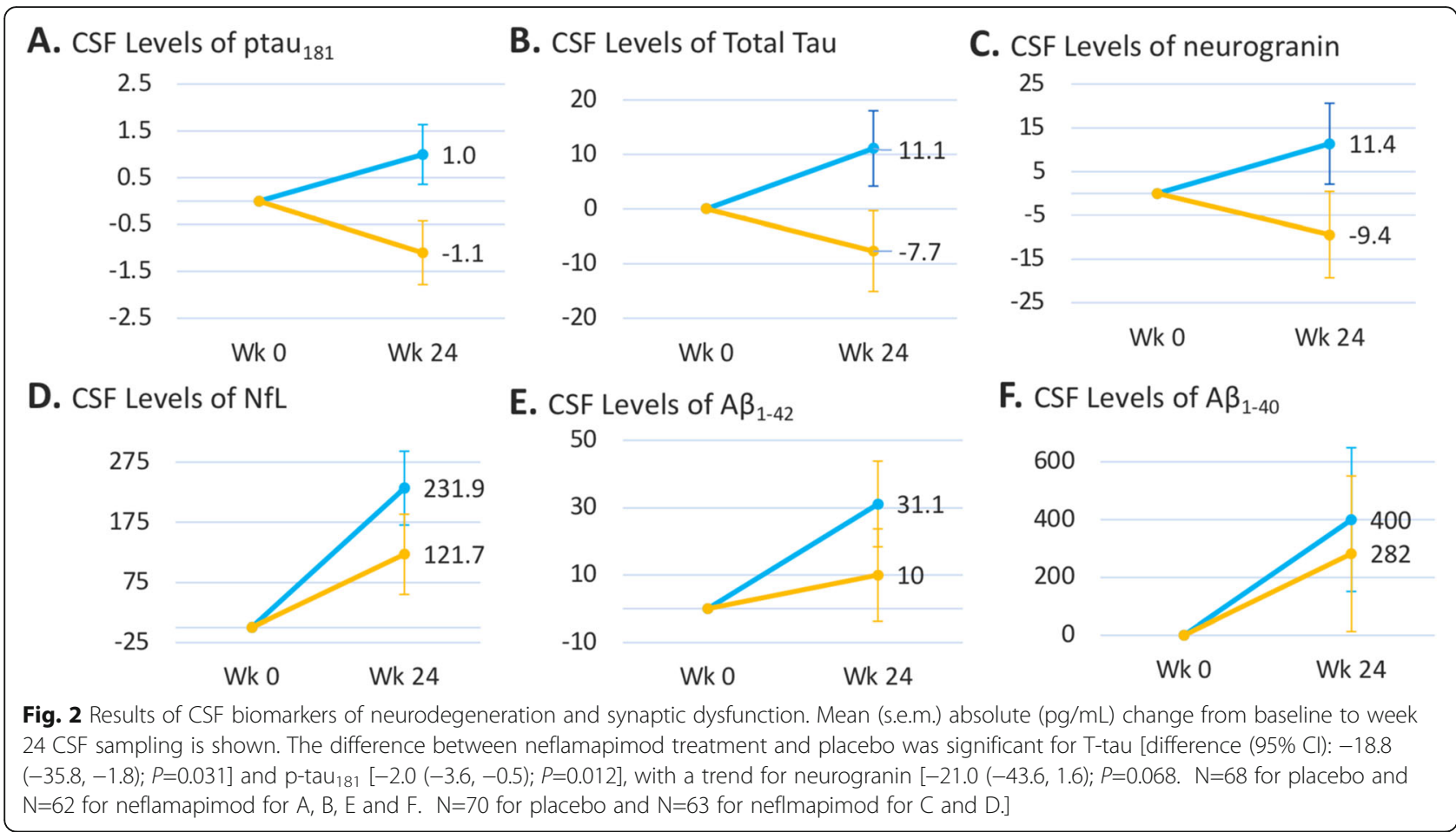

worsening in WMS Immediate and Delayed Recall, particularly the subjects on background $\mathrm{AD}$ therapy. In the prespecified analysis, no differences in the outcome on WMS Immediate and Recall were noted in neflamapimod subjects with $C_{\text {trough }}>75$ th percentile compared with placebo recipients in the overall patient population (Supplemental Figure 2a). However, in an exploratory analysis, within those receiving background AD therapy, there were positive trends in the outcome on WMS Immediate and Delayed Recall, favoring neflamapimod subjects with $\mathrm{C}_{\text {trough }}>75$ th percentile compared with placebo recipients (Supplemental Figure 2b). In additional exploratory analyses, neflamapimod subjects with the highest $\mathrm{C}_{\text {trough }}$ drug levels also showed minor trends towards less worsening of both the MMSE and CDR-SB, compared with the placebo group (Supplemental Figure 3).

Table 3 shows the effects on CSF biomarkers by $\mathrm{C}_{\text {trough }}$ in neflamapimod subjects compared with placebo, utilizing the pre-specified cut-off of 75 th percentile for $\mathrm{C}_{\text {trough }}$ in neflamapimod subjects. For T-tau, p-tau ${ }_{181}$ and $\mathrm{A} \beta_{42}$ a significant $(p<0.05)$ plasma trough dependent effect was evident. Only minor or no trends were evident in the other 3 CSF biomarkers, perhaps due to the greater within-subject variability in change for these three biomarkers compared with the first three.

\section{Safety}

Neflamapimod was well tolerated in this trial. Adverse events (AEs) occurring at $\geq 5 \%$ incidence in the neflamapimod group were fall (6\% in neflamapimod, $4 \%$ in placebo), headache $(6 \%, 4 \%)$, diarrhea $(5 \%, 2 \%)$, and upper respiratory tract infection $(5 \%, 8 \%)$. No on-study deaths were reported. Serious AEs (SAEs) reported in the neflamapimod group were hypokalemia and plasma cell myeloma (in different subjects), both considered unrelated to treatment. AEs leading to study drug discontinuation, each reported by 1 subject, included nausea and plasma cell myeloma (also an SAE) in the neflamapimod group and a fall leading to a subdural hematoma in the placebo group. Though analyses of group mean changes in alanine and aspartate aminotransferase levels did not reveal any differences between neflamapimod and placebo, one neflamapimod-treated subject had elevations in these enzymes to approximately 3 times the upper-limit-of-normal (ULN), which started to resolve within 1 week after treatment cessation, at which time the patient withdrew from the trial.

\section{Discussion}

The major objective of this multi-center phase 2, randomized, double-blind placebo-controlled 24-week trial was to determine whether p38 $\alpha$ inhibition could reverse synaptic dysfunction and, with it restore function, at least partially, as assessed by episodic memory performance. Toward that objective, in the primary endpoint, we found no difference between neflamapimod treatment and placebo in changes in episodic memory performance, as measured with the HVLT-R. However, the CSF biomarker results provide evidence suggesting that neflamapimod treatment had intended biological effects. In 
A.

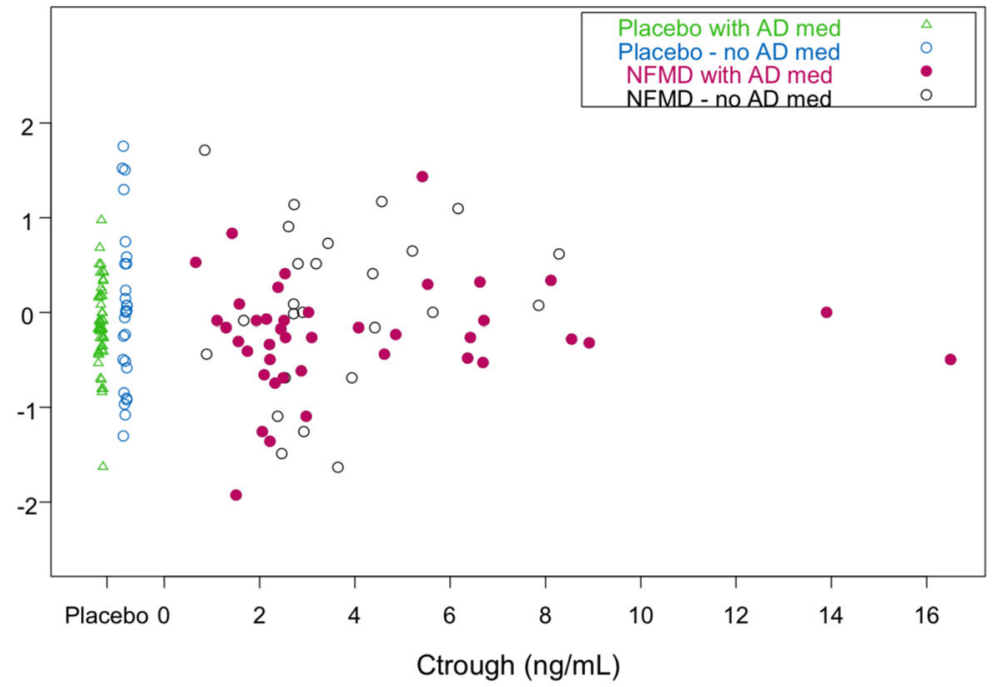

B.

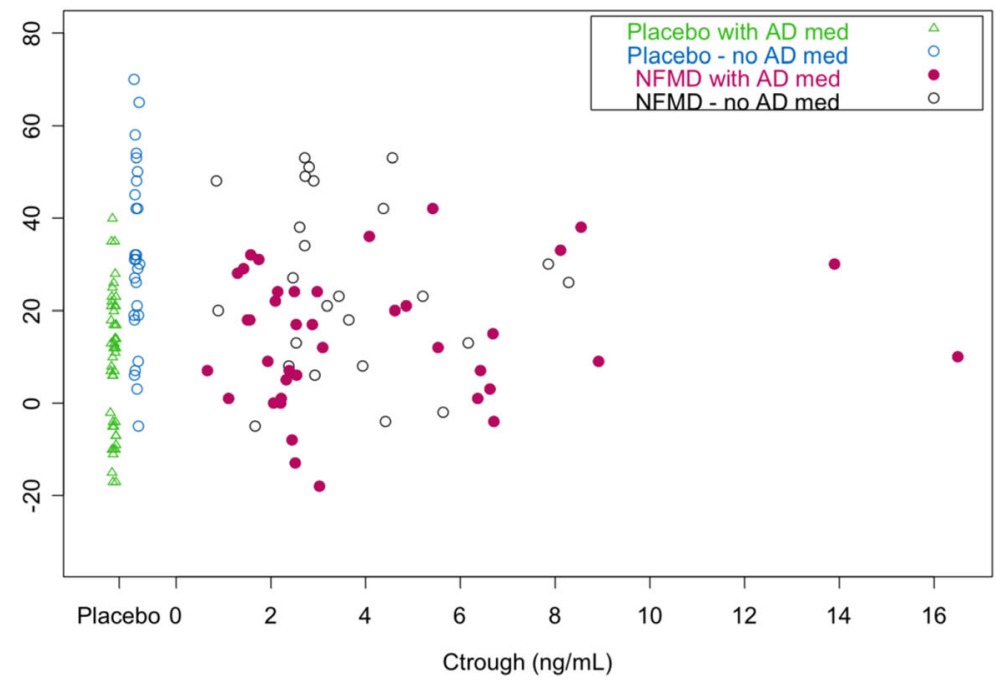

Fig. 3 Relationship between $C_{\text {trough }}$ and episodic memory measures. Plasma trough drug concentration in neflamapimod versus change from baseline to week 24. a HVLT-R combined total and delayed recall Z-score (i.e., primary endpoint), and $\mathbf{b}$ WMS combined immediate and delayed recall composite is shown in circles (open for treatment-naïve subjects, closed for those on background AD therapy). For comparison, placebo subjects are shown in triangles (open for treatment-naïve subjects, closed for those on background AD therapy) on the left side of the figure

Table 3 Median (range) change from baseline to week 24 in plasma biomarkers by $C_{\text {trough }}$ plasma neflamapimod levels on day 21 versus placebo

\begin{tabular}{|c|c|c|c|c|}
\hline \multirow[t]{2}{*}{ Biomarker } & \multirow{2}{*}{$\begin{array}{l}\text { Placebo }(N= \\
67)\end{array}$} & \multicolumn{2}{|l|}{ Neflamapimod by $C_{\text {trough }}$} & \multirow{2}{*}{$\begin{array}{l}P \text { value for } \\
\text { downward } \\
\text { trend }^{1}\end{array}$} \\
\hline & & $\leq 75 \%$ Percentile $(N=42)$ & $>75 \%$ Percentile $(N=13)$ & \\
\hline Total tau & $7.0(-84.2,489.2)$ & $2.9(-68.4,48.4)$ & $3.6(-30.7,22.8)$ & 0.04 \\
\hline P-tau 181 & $0.67(-6.4,44.0)$ & $0.03(-5.5,6.8)$ & $0.06(-4.2,3.0)$ & 0.02 \\
\hline$A \beta_{1-40}$ & $221(-6753,4988)$ & $465(-6017,8524)$ & $126(-2070,2568)$ & 0.24 \\
\hline$A \beta_{1-42}$ & $-1.6(-150,268)$ & $5.5(-298,205)$ & $-37.7(-136,92)$ & 0.05 \\
\hline Neurogranin & $9.1(-128,481)$ & $-0.65(-124,323)$ & $11.3(-44,75)$ & 0.1 \\
\hline Neurofilament light chain & $60(-655,3933)$ & $42(-1417,513)$ & $53(-322,665)$ & 0.16 \\
\hline
\end{tabular}

${ }^{1}$ Jonckheere-Terpstra test for downward trend of change from baseline for 3 groups: placebo, $C_{\text {trough }} \leq 75$ th percentile and $C_{\text {trough }}>75$ th percentile 
particular, significantly decreased levels of the disease biomarkers CSF T-tau and CSF p-tau ${ }_{181}$ were observed with neflamapimod, compared with placebo, over the 24-week treatment period in the full efficacy population. For both $\mathrm{T}$-tau and $\mathrm{p}$-tau ${ }_{181}$, there was a mean $\sim 3 \%$ increase in CSF levels in the placebo group, as expected, while there was a decrease of similar magnitude in the neflamapimod group. Tau phosphorylation and tau pathology have been identified in preclinical studies as downstream consequences of abnormally high p38 $\alpha$ kinase activity $[10,16,20]$. Further, neflamapimod treatment in the Ts2 transgenic mouse model decreased, relative to vehicle, levels of p-tau (pS202) in the brain (Nixon RA, personal communication; presentation at Alzheimer's Disease Genetics Global Symposium, 22 September 2020). Thus, as the reduction in tau phosphorylation is a consequence of $\mathrm{p} 38 \alpha$ inhibition, the decreases in p-tau and tau in CSF in the current study demonstrate, at a minimum, target engagement for neflamapimod with respect to $\mathrm{p} 38 \alpha$ inhibition. In addition, p-tau, tau, and neurogranin are the protein markers generally considered to be most closely associated with synaptic dysfunction in AD [34-40], suggesting that neflamapimod has a beneficial effect on synaptic dysfunction.

Results of the pre-specified PK-PD analyses suggest that a major factor in not meeting the primary clinical objective, despite significant effects of neflamapimod treatment on CSF biomarker levels, is that the dose of $40 \mathrm{mg}$ twice daily was too low. Specifically, for the primary endpoint, HVLT-R, there were trends towards improvement, relative to placebo, from baseline to week 24 in subjects with either $C_{\text {trough }} \geq 4 \mathrm{ng} / \mathrm{mL}$ or by the pre-specified cut-off for analysis of 75th percentile for $\mathrm{C}_{\text {trough }}$ within the study (5.4 $\mathrm{ng} / \mathrm{mL}$ ). This observation was supported by results in the secondary measure of episodic memory, WMS Immediate and Delayed Recall composites, in subjects on background AD therapy. Among these, neflamapimod-treated subjects having $\mathrm{C}_{\text {trough }}$ levels above the pre-specified 75th percentile demonstrated significantly better outcomes on WMS Immediate and Delayed at weeks 12 and 24, than did those on placebo. The 4 to $5 \mathrm{ng} / \mathrm{mL}$ threshold is consistent with current understanding of the mechanism-ofaction and potency of neflamapimod. When the trial was designed, there were no potency data available for neflamapimod in AD-relevant pharmacology; instead, the dose level of $40 \mathrm{mg}$ was chosen based on effective doses of neflamapimod in aged rats [18]. Recent mechanistic studies indicate that a major pharmacological target of neflamapimod is endolysosomal dysfunction associated with the protein Rab5 [41]. In addition, neflamapimod was shown to block $A \beta$ oligomer-induced, as well prioninduced, dendritic spine loss in hippocampal neurons [42]. The in vitro potency, $\mathrm{EC}_{50}$, of neflamapimod for reversing Rab5+ endolysosomal dysfunction and for blocking $A \beta$-oligomer or prion-induced dendritic spine loss is $5-12.5 \mathrm{ng} / \mathrm{mL}$. As brain concentrations of neflamapimod in preclinical studies are approximately two-fold higher than in the plasma, those potency concentrations are overlapping with predicted brain concentrations based on a plasma drug concentration of 4 to $5 \mathrm{ng} / \mathrm{mL}$.

The trial was designed and powered with a hypothesis, based results in the preclinical models and in the two phase 2a clinical trials, that there would be substantial improvement from baseline in episodic memory function within the neflamapimod treatment group. The sample size in the current trial was based on the results of the phase $2 \mathrm{a}$ trials where, in both the 16-patient, 12-week trial (Study 302) that assessed episodic memory with the WMS and the 9-patient, 6-week trial (Study 303) that assessed episodic memory with the HVLT-R, the effect size for improvement from baseline exceeded 0.6. Based on those results, the sample size for the current study provided $>80 \%$ statistical power for an effect size of 0.45 for the difference between neflamapimod treatment and placebo. Thus, with the decline of 0.15 from baseline to week 24 in the HVLT-R $Z$-score in the placebo-group in the current trial, an improvement from baseline to week 24 within the neflamapimod treatment group of at least 0.3 in the HVLT-R $A$-score of 0.3 was required to demonstrate a statistically significant effect on the primary endpoint, an effect not seen. This difference in outcome between the current trial and the two earlier phase $2 \mathrm{a}$ studies may be related to the two major differences between the current trial and phase 2a: (1) the patients in the earlier studies were less advanced in the disease and not receiving background AD therapy, and (2) the phase 2a studies included a higher dose of neflamapimod and achieved higher blood drug concentration levels. An impact of background therapy on the outcome is suggested by the neflamapimod participants not on background therapy with $C_{\text {trough }} \geq 75$ th percentile having an improvement from baseline of $0.45 \mathrm{Z}$-score on the primary endpoint (see Supplemental Figure 1). However, there were only five participants included in this analysis and, so, no conclusions can be drawn from it. With regard to the doses utilized in phase 2a, in Study 302 that utilized the WMS, nine participants received the same $40-\mathrm{mg}$ BID dose as in the current study and seven received 125-mg BID (note: due to differences in excipient ratios utilized in the drug capsules, the $125 \mathrm{mg}$ resulted in plasma drug levels, on average, only $50 \%$ higher than with 40-mg BID). Within Study 302, a statistically significant PK-PD relationship was established [27], a relationship indicating that plasma drug levels resulting from 125-mg BID were associated with greater improvement in WMS scores. In retrospect, and after obtaining the WMS results in the current study, the 
"improvement" seen in the $40 \mathrm{mg}$ BID group likely resulted primarily from practice/learning effects, though the additional improvement at 50\% higher plasma drug levels may still have been related to neflamapimod treatment. In the other phase 2a study (Study 303), in which positive effects on HVLT-R were demonstrated, the average plasma drug exposure with 40-mg BID was approximately $50 \%$ higher than in the current study because extremely low weight subjects were included. We believe that the results in Study 303 were not due to practice effects, as there were no practice/learning effects evident on the HVLT-R in the current study, including in the participants not receiving background therapy. Overall, though limited by the small number of participants in the phase 2a studies, the plasma drug concentration-effect relationships in phase $2 \mathrm{a}$ are generally consistent with the PK-PD analyses in the current study and suggest that the therapeutically active dose range is at least $50 \%$ higher than $40-\mathrm{mg}$ BID (e.g., $\geq 40$-mg TID).

With a specific kinase inhibitor, where both biomarker and clinical effects would depend on inhibition of that target kinase, the expectation would be that the dose-response would be similar for the biomarker and clinical effects. In this case, the differences between neflamapimod and placebo effects on the biomarkers were modest $(\sim 5 \%)$ which, in the absence of a clinical effect, suggests that the 40-mg BID dose level is simply at the lower end of the pharmacologically active dose range. Indeed, biomarkers are, by design, intended to be more sensitive than clinical effects and it is not unusual to see biomarker effects preceding clinical effects with respect to dose (i.e. at a lower dose) and/or time. For example, with aducanumab, significant effects on brain amyloid plaque by PET scan were seen at $3 \mathrm{mg} / \mathrm{kg}$, though the clinical effects are limited to a dose of $10 \mathrm{mg} / \mathrm{kg}$, where a moderately greater effect on amyloid plaque reduction is also seen [43]. Furthermore, at the clinically efficacious 10 $\mathrm{mg} / \mathrm{kg}$ dose level, the majority of the effect on amyloid plaque load is seen by 26 weeks, while the clinical effect is not evident until week 52. To determine whether such relationships between biomarker and clinical effects exist for neflamapimod in $\mathrm{AD}$ will require further upward dose-ranging to first establish a clinical effect. In addition, a longer duration clinical trial may show a more distinct plasma drug concentration-effect relationship (i.e., dose-response), in terms of CSF biomarker effects, than we were able to demonstrate in the current study.

Non-CNS AEs, particularly aminotransferase elevations, have limited development of p38 MAPK inhibitors for peripheral inflammatory disorders [44]. Neflamapimod has the potential to minimize such toxicities, while maintaining robust pharmacological effects in the brain.
The reasons for this include that plasma drug concentrations are half that in the brain and the drug is 95\% protein-bound in whole blood, further reducing peripheral effects, as protein-binding decreases its potency three-fold. Our results support this concept, as only one of 78 neflamapimod recipients developed aminotransferase elevation to 3 times ULN, while pharmacological activity was demonstrated by the CSF biomarker results. Further, as the incidence of aminotransferase levels $\geq 3$ times ULN was approximately $15 \%$ in a prior study of neflamapimod in rheumatoid arthritis patients, at a dose of $250 \mathrm{mg}$ twice daily $\left(\mathrm{C}_{\text {trough }}\right.$ approximately $\left.30 \mathrm{ng} / \mathrm{mL}\right)$ [44], a low incidence of liver enzyme elevation is expected with dosing regimens that would consistently achieve $\mathrm{C}_{\text {trough }} \geq 4 \mathrm{ng} / \mathrm{mL}$.

\section{Limitations}

This trial has limitations. First, the 24-week duration of the trial was not designed to ascertain effects on clinical disease progression. The sample size was effectively further attenuated because only a minority of subjects achieved plasma drug levels in the identified potentially therapeutically active range. In the PK-PD analysis, the sparse sampling approach utilized did not provide sufficient information to develop a robust population PK model, which would have allowed for a more thorough evaluation of the relationship between outcomes and PK parameters other than $\mathrm{C}_{\text {trough }}$. Our two measures of episodic memory each has its respective strengths and limitations. The WMS as a composite of three different cognitive tests provides the more comprehensive assessment of memory function. In addition, having three modestly correlated cognitive tests inherently decreases variability for the composite assessment. However, with the repeated application of the WMS, which has no alternative versions (that is, the same version is applied at each visit), over the relatively short-time period of the study, we saw substantial practice effects in treatment naïve patients that precluded any ability to discern neflamapimod effects. With the HVLT-R, which has alternative versions, at a group level, there were very little practice effects, as there was very little change in mean HVLT-R Z-scores over the 24 weeks of the study. However, with a single test, there was substantial withinsubject variability from visit to visit. For example, within the placebo group, from baseline to week 6 , nearly all scores for subjects above the median at baseline decreased, while those for all the subjects below the median increased; that is, there was substantial regression to mean. One approach to handling such variability would be to have more than one assessment at baseline, for example, one during the first screening visit and one on day 1 before starting treatment. The optimal approach would be to have a cognitive testing battery 
composed of three or more distinct episodic memory tests, each with alternate versions.

\section{Conclusions}

This clinical trial did not show an effect of improving episodic memory function with 40-mg neflamapimod twice daily for 24 weeks in patients with mild AD. As the PK-PD analyses suggest that neflamapimod-treated subjects with higher plasma levels showed less episodic memory decline, an insufficient dose may have contributed to the lack of clinical efficacy. However, at this dose level, neflamapimod treatment modestly decreased CSF protein markers of synaptic dysfunction relative to placebo treatment. The results indicate that a longer duration clinical trial of neflamapimod at a higher dose level to assess effects on $\mathrm{AD}$ progression is warranted.

\section{Supplementary Information}

The online version contains supplementary material available at https:/doi. org/10.1186/s13195-021-00843-2.

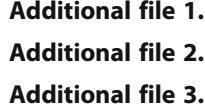

\section{Acknowledgements}

REVERSE-SD Clinical Sites (Site principal investigator): USA: Massachusetts General Hospital (Arnold); CITrials, Inc (Coskinas); Florida Premier Research Institute (Gonzales); Manhattan Behavioral Medicine LLC (Joseph); Northwest Clinical Research Center (Khan); Northwest Clinical Trials (McConnehey); Miami Dade Medical Research Institute (Paricio); Sensible Healthcare LLC (Taylor); Alliance Research (Zarate Rowell); Anchor Neuroscience (Groom); Southern California Research LLC (Summers); Progressive Medica Research (White); Pacific Research Network, LLC (Thein); Alzheimer's Memory Center (Bolouri); Viking Clinical Research, Ltd (Schwartz); Suncoast Neuroscience Associate (Vasquez). UK: MAC Clinical Research Manchester (Asher); MAC Clinical Research Leeds (Ball); MAC Clinical Research Liverpool (Munthali); MAC Clinical Research Blackpool (Kabir); St Pancras Clinical Research (Langford); S Boroughs/North West Boroughs Healthcare (Komuravelli); Clinical Research Tankerlsey (Lynch); Cognition Health Ltd (MacSweeney); Cognition Health Ltd (Pearson); Cognition Health Birmingham (Faulkner); Cognition Health Ltd (Miller); Fulbourn Hospital (Underwood). Netherlands: Brain Research Center (Prins); Jeroen Bosch Ziekenhuis (Dautzenberg); Amphia Ziekhuis (Van Norden). Czech Republic: Vestra Clinics s.r.o. (Pazdera); Cerbrovaskularni poradn s.r.o. (Bar) Private Psychiatric Centre (Dvacka); Neruo HK s.r.o., Poliklinika Chocen (Valis); Clintrial s.r.o. (Votypkova). Denmark: CCBR Clinical Research, Aalborg (Areovimata); CCBR Clinical Research, Vejle (Justesen); and CCBR Clinical Research, Ballerup (Schmidt).

We are very grateful to all patients and caregivers who participated in this study and to the many staff members at the clinical sites for their dedication and commitment to the study. We also acknowledge the study project teams at EIP Pharma and WCT, and specifically, the excellent leadership of those project teams by Jennifer Conway and Nadia Bodjarian, respectively, as well the tremendous contributions of Natalia Drosopolou (project supervision) and Shuhua Qi (statistics) at WCT.

\section{Authors' contributions}

JJA and HMC had full access to all of the data and take responsibility for the integrity of the data and accuracy of the data analysis. Concept and design: JJA, JEH, NDP, and PS. Acquisition, analysis, or interpretation of data: All authors. Drafting of the manuscript: JJA, NDP, and PS. Critical revision of the manuscript for important intellectual content: JJA, JEH, NDP, and PS. Statistical analysis: HMC and JJA. Obtained funding: JJA, KB. Administrative, technical, or material support: JJA, KB, JEH, NDP, and PS. Supervision: Alam and Scheltens. The authors read and approved the manuscript.

\section{Authors' information}

Not applicable.

\section{Funding}

The trial was sponsored and funded by EIP Pharma, Inc., which also provided the study drug. NDP and PS were compensated for their time towards protocol development, participation in joint project team and study steering committee meetings, and for manuscript preparation. HMC and JEH were engaged as consultants by EIP Pharma, Inc. JJA and KB are employees of EIP Pharma, Inc.

The sponsor, EIP Pharma, Inc., conceived of and designed the trial in collaboration with PS, NDP, and JEH. EIP Pharma then engaged and managed the Worldwide Clinical Trials (WCT) to conduct the trial through the clinical trial investigator sites, collect and manage data, and conduct primary statistical analyses. Employees of EIP Pharma (JJA, KB) were involved in the preparation and review of the manuscript and the decision to submit the manuscript.

\section{Availability of data and materials}

Clinical and biomarker endpoint datasets will be made available upon reasonable request to the sponsor, EIP Pharma.

\section{Declarations}

\section{Ethics approval and consent to participate}

Ethics approval was obtained and participants provided written consent prior to the any study procedures. Ethics committees that approved the study are as follows:

- USA: Copernicus Group Independent Review Board (CGIRB), Cary NC (IRB reference number IRB00001313)

- UK: REC- London - Riverside, Chelsea \& Westminster Hospital, London - Netherlands: Foundation Beoordeling Ethiek Biomedisch Onderzoek (Foundation BEBO), Assen

- Denmark: Den Videnskabsetiske Komité for Region Nordjylland, Aalborg Øst - Czech Republic: Eticka Komise Vestra Clinics, Rychnov nad Kněžnou; Etická komise nestátního zdravotnického zařizení CLINTRIAL, s.r.o., Praha

\section{Consent for publication}

Not applicable.

\section{Competing interests}

NDP is a consultant to Boehringer Ingelheim. He serves on the DSMB of Abbvie's M15-566 trial. He is the CEO and co-owner of the Brain Research Center, The Netherlands.

JEH reports receipt of personal fees in the past 2 years from AlzeCure, Aptinyx, Astra Zeneca, Athira Therapeutics, Axon Neuroscience, Axovant, Biogen Idec, BlackThornRx, Boehringer Ingelheim, Cerecin, Cognition Therapeutics, Compass Pathways, CRF Health, Curasen, EIP Pharma, Eisai, Eli Lilly, FSV7, G4X Discovery, GfHEU, Heptares, Lundbeck, Lysosome Therapeutics, MyCognition, Neurocentria, Neurocog, Neurodyn Inc, Neurotrack, Novartis, Nutricia, Probiodrug, Regeneron, Rodin Therapeutics, Samumed, Sanofi, Servier, Signant, Syndesi Therapeutics, Takeda, Vivoryon Therapeutics, vTv Therapeutics, and Winterlight Labs. Additionally, he holds stock options in Neurotrack Inc. and is a joint holder of patents with My Cognition Ltd.

$\mathrm{H}-\mathrm{MC}$ has no conflicts to declare. JJA is the founder and CEO and Ms. Kelly Blackburn is an employee of the sponsor of the trial, EIP Pharma Inc.

PS reports receipt of fees (to the university) in the past 2 years from Axon Neuroscience, Cognition Therapeutics, EIP Pharma, Green Valley, GemVax, Novartis, Vivoryon (Probiodrug AG), FUJ Film/Toyama, Rodin Therapeutics, and Medavante. He is a paid medical advisor to the Brain Research Center and serves on the supervisory board.

\section{Author details}

${ }^{1}$ Alzheimer Center, Department of Neurology, Amsterdam UMC, Amsterdam, The Netherlands. ${ }^{2}$ Brain Research Center, Amsterdam, The Netherlands. ${ }^{3}$ Institute of Psychiatry, Psychology \& Neuroscience, King's College London, 
London, UK. ${ }^{4}$ Metis Cognition Ltd., Wiltshire, UK. ${ }^{5}$ Anoixis Corporation, Natick, MA, USA. ${ }^{6}$ EIP Pharma, Inc., Boston, MA, USA.

\section{Received: 24 January 2021 Accepted: 4 May 2021 Published online: 27 May 2021}

\section{References}

1. Chen Y, Fu AKY, Ip NY. Synaptic dysfunction in Alzheimer's disease: mechanisms and therapeutic strategies. Pharmacol Ther. 2019;195:186-98. https://doi.org/10.1016/j.pharmthera.2018.11.006.

2. Jackson J, Jambrina E, Li J, Marston H, Menzies F, Phillips K, et al. Targeting the synapse in Alzheimer's disease. Front Neurosci. 2019;13:735. https://doi org/10.3389/fnins.2019.00735.

3. Pei YA, Davies J, Zhang M, Zhang HT. The role of synaptic dysfunction in Alzheimer's disease. J Alzheimers Dis. 2020;76(1):49-62. https://doi.org/1 0.3233/JAD-191334.

4. Freeman OJ, Mallucci GR. The UPR and synaptic dysfunction in neurodegeneration. Brain Res. 2016;1648(Pt B):530-7.

5. Kheiri G, Dolatshahi M, Rahmani F, Rezaei N. Role of p38/MAPKs in Alzheimer's disease: implications for amyloid beta toxicity targeted therapy. Rev Neurosci. 2018;30(1):9-30. https://doi.org/10.1515/revneuro-2018-0008.

6. Lee JK, Kim NJ. Recent advances in the inhibition of p38 MAPK as a potential strategy for the treatment of Alzheimer's disease. Molecules. 2017; 22(8). https://doi.org/10.3390/molecules22081287.

7. Gee MS, Son SH, Jeon SH, Do J, Kim N, Ju YJ, et al. A selective p38alpha/ beta MAPK inhibitor alleviates neuropathology and cognitive impairment, and modulates microglia function in 5XFAD mouse. Alzheimers Res Ther. 2020;12(1):45. https://doi.org/10.1186/s13195-020-00617-2.

8. Correa SA, Eales KL. The role of p38 MAPK and its substrates in neuronal plasticity and neurodegenerative disease. J Signal Transduct. 2012;2012:112. https://doi.org/10.1155/2012/649079.

9. Yasuda S, Sugiura H, Tanaka H, Takigami S, Yamagata K. p38 MAP kinase inhibitors as potential therapeutic drugs for neural diseases. Cent Nerv Syst Agents Med Chem. 2011;11(1):45-59. https://doi.org/10.2174/1 87152411794961040.

10. Li Y, Liu L, Barger SW, Griffin WS. Interleukin-1 mediates pathological effects of microglia on tau phosphorylation and on synaptophysin synthesis in cortical neurons through a p38-MAPK pathway. J Neurosci. 2003;23(5):160511. https://doi.org/10.1523/JNEUROSCI.23-05-01605.2003.

11. Prieto GA, Snigdha S, Baglietto-Vargas D, Smith ED, Berchtold NC, Tong L, et al. Synapse-specific IL-1 receptor subunit reconfiguration augments vulnerability to IL-1 beta in the aged hippocampus. Proc Natl Acad Sci U S A. 2015;112(36):E5078-87. https://doi.org/10.1073/pnas.1514486112.

12. Li S, Jin M, Koeglsperger T, Shepardson NE, Shankar GM, Selkoe DJ. Soluble Abeta oligomers inhibit long-term potentiation through a mechanism involving excessive activation of extrasynaptic NR2B-containing NMDA receptors. J Neurosci. 2011;31(18):6627-38. https://doi.org/10.1523/ JNEUROSCI.0203-11.2011.

13. Watterson DM, Grum-Tokars VL, Roy SM, Schavocky JP, Bradaric BD, Bachstetter AD, et al. Development of novel in vivo chemical probes to address CNS protein kinase involvement in synaptic dysfunction. PLoS One. 2013;8(6):e66226. https://doi.org/10.1371/journal.pone.0066226.

14. Birnbaum JH, Bali J, Rajendran L, Nitsch RM, Tackenberg C. Calcium fluxindependent NMDA receptor activity is required for Abeta oligomerinduced synaptic loss. Cell Death Dis. 2015;6(6):e1791. https://doi.org/10.103 8/cddis.2015.160.

15. Koppensteiner P, Trinchese F, Fa M, Puzzo D, Gulisano W, Yan S, et al. Timedependent reversal of synaptic plasticity induced by physiological concentrations of oligomeric Abeta42: an early index of Alzheimer's disease. Sci Rep. 2016;6:32553.

16. Bhaskar K, Konerth M, Kokiko-Cochran ON, Cardona A, Ransohoff RM, Lamb BT. Regulation of tau pathology by the microglial fractalkine receptor. Neuron. 2010;68(1):19-31. https://doi.org/10.1016/..neuron.2010.08.023.

17. Lauretti E, Li JG, Di Meco A, Pratico D. Glucose deficit triggers tau pathology and synaptic dysfunction in a tauopathy mouse model. Transl Psychiatry. 2017;7(1):e1020. https://doi.org/10.1038/tp.2016.296.

18. Alam JJ. Selective brain-targeted antagonism of p38 MAPKalpha reduces hippocampal IL-1 beta levels and improves Morris water maze performance in aged rats. J Alzheimers Dis. 2015:48(1):219-27. https://doi.org/10.3233/JA D-150277.
19. Roy SM, Grum-Tokars VL, Schavocky JP, Saeed F, Staniszewski A, Teich AF, et al. Targeting human central nervous system protein kinases: an isoform selective p38alphaMAPK inhibitor that attenuates disease progression in Alzheimer's disease mouse models. ACS Chem Neurosci. 2015;6(4):666-80. https://doi.org/10.1021/acschemneuro.5b00002.

20. Maphis N, Jiang S, Xu G, Kokiko-Cochran ON, Roy SM, Van Eldik L, et al. Selective suppression of the alpha isoform of p38 MAPK rescues late-stage tau pathology. Alzheimers Res Ther. 2016;8(1):54. https://doi.org/10.1186/ s13195-016-0221-y.

21. Colie S, Sarroca S, Palenzuela R, Garcia I, Matheu A, Corpas R, et al. Neuronal p38alpha mediates synaptic and cognitive dysfunction in an Alzheimer's mouse model by controlling beta-amyloid production. Sci Rep. 2017;7(1): 45306. https://doi.org/10.1038/srep45306.

22. Schnoder L, Hao W, Qin Y, Liu S, Tomic I, Liu X, et al. Deficiency of neuronal p38alpha MAPK attenuates amyloid pathology in Alzheimer disease mouse and cell models through facilitating lysosomal degradation of BACE1. J Biol Chem. 2016:291 (5):2067-79. https://doi.org/10.1074/jbc.M115.695916.

23. Cortez I, Bulavin DV, Wu P, McGrath EL, Cunningham KA, Wakamiya M, et al. Aged dominant negative p38alpha MAPK mice are resistant to agedependent decline in adult-neurogenesis and context discrimination fear conditioning. Behav Brain Res. 2017;322(Pt B):212-22.

24. Moreno-Cugnon L, Revuelta M, Arrizabalaga O, Colie S, Moreno-Valladares M, Jimenez-Blasco D, et al. Neuronal p38alpha mediates age-associated neural stem cell exhaustion and cognitive decline. Aging Cell. 2019;18(6): e13044. https://doi.org/10.1111/acel.13044.

25. Huentelman MJ, Piras IS, Siniard AL, De Both MD, Richholt RF, Balak CD, et al. Associations of MAP2K3 gene variants with superior memory in superagers. Front Aging Neurosci. 2018;10:155. https://doi.org/10.3389/fna gi.2018.00155.

26. Navarrete M, Cuartero MI, Palenzuela R, Draffin JE, Konomi A, Serra I, et al. Astrocytic p38alpha MAPK drives NMDA receptor-dependent long-term depression and modulates long-term memory. Nat Commun. 2019;10(1): 2968. https://doi.org/10.1038/s41467-019-10830-9.

27. Scheltens $\mathrm{P}$, Prins $\mathrm{N}$, Lammertsma A, Yaqub M, Gouw A, Wink AM, et al. An exploratory clinical study of p38alpha kinase inhibition in Alzheimer's disease. Ann Clin Transl Neurol. 2018;5(4):464-73. https://doi.org/10.1002/a cn3.549.

28. Alam J, Gevorkyan H, Jhee S, Park L, Ki J-H, Alaka N, et al. Clinical pharmacology study of p38 alpha MAP kinase inhibitor, neflamapimod (VX745 ) in mild cognitive impairment (MCI) due to Alzheimer's disease (AD) or mild AD. J Prev Alzheimers Dis. 2016;3(Suppl 1):277.

29. International Council for Harmonisation of Technical Requirements for Pharmaceuticals for Human Use. ICH Harmonized Guideline. Integrated Addendum to ICH E6(R1). Guideline for Good Clinical Practice E6(R2) 2016 https://database.ich.org/sites/default/files/E6_R2_Addendum.pdf. Accessed 24 Apr 2020.

30. World-Medical-Association. World Medical Association Declaration of Helsinki: ethical principles for medical research involving human subjects. JAMA. 2013;310(20):2191-4. https://doi.org/10.1001/jama.2013.281053.

31. Shapiro AM, Benedict RH, Schretlen D, Brandt J. Construct and concurrent validity of the Hopkins Verbal Learning Test-revised. Clin Neuropsychol. 1999;13(3):348-58. https://doi.org/10.1076/clin.13.3.348.1749.

32. Maccow G. WMS-IV: Administration, Scoring, Basic Interpretation 2011 http://images.pearsonclinical.com/images/Products/WMS-IV/WMS-IV_Webina r_September_2011_Handout.pdf. Accessed 24 Apr 2020.

33. Charles River Laboratories Edinburgh Ltd. Technical Report, Study Number 322119. Validation of analytical procedures for the determination of VX-745 in human plasma and cerebrospinal fluid by LC-MS/MS. Written in 2015. On file at EIP Pharma, Inc.

34. Hoover BR, Reed MN, Su J, Penrod RD, Kotilinek LA, Grant MK, et al. Tau mislocalization to dendritic spines mediates synaptic dysfunction independently of neurodegeneration. Neuron. 2010;68(6):1067-81. https:// doi.org/10.1016/..neuron.2010.11.030.

35. Coomans EM, Schoonhoven DN, Tuncel H, Verfaillie SCJ, Wolters EE, Boellaard R, et al. In vivo tau pathology is associated with synaptic loss and altered synaptic function. Alzheimers Res Ther. 2021;13(1):35. https://doi. org/10.1186/s13195-021-00772-0.

36. Mila-Aloma M, Salvado G, Gispert JD, Vilor-Tejedor N, Grau-Rivera O, Sala-Vila A, et al. Amyloid beta, tau, synaptic, neurodegeneration, and glial biomarkers in the preclinical stage of the Alzheimer's continuum. Alzheimers Dement. 2020;16(10):1358-71. https://doi.org/10.1002/alz.12131. 
37. Casaletto KB, Elahi FM, Bettcher BM, Neuhaus J, Bendlin BB, Asthana S, et al. Neurogranin, a synaptic protein, is associated with memory independent of Alzheimer biomarkers. Neurology. 2017;89(17):1782-8. https://doi.org/1 0.1212 WNL.00000000000004569.

38. Zhou L, McInnes J, Wierda K, Holt M, Herrmann AG, Jackson RJ, et al. Tau association with synaptic vesicles causes presynaptic dysfunction. Nat Commun. 2017;8(1):15295. https://doi.org/10.1038/ncomms15295.

39. Mattsson $\mathrm{N}$, Insel PS, Palmqvist $\mathrm{S}$, Portelius $\mathrm{E}$, Zetterberg $\mathrm{H}$, Weiner $\mathrm{M}$, et al Cerebrospinal fluid tau, neurogranin, and neurofilament light in Alzheimer's disease. EMBO Mol Med. 2016;8(10):1184-96. https://doi.org/10.15252/ emmm.201606540

40. Kim WH, Racine AM, Adluru N, Hwang SJ, Blennow K, Zetterberg H, et al. Cerebrospinal fluid biomarkers of neurofibrillary tangles and synaptic dysfunction are associated with longitudinal decline in white matter connectivity: a multi-resolution graph analysis. Neuroimage Clin. 2019;21 101586. https://doi.org/10.1016/j.nicl.2018.10.024.

41. Jiang Y, Stavrides P, Darii S, Yang D, Bleiwas C, Smiley J, et al. Effects of p38a MAP kinase inhibition on the neurodegenerative phenotype of the Ts2 Down Syndrome mouse model. Alzheimers Dement. 2019;15(7, Suppl): P1597.

42. Amin L, Le N, Mercer RCC, Germann U, Alam J, Harris DA. Role of p38a MAP kinase in amyloid- $\beta$ derived diffusible ligand (ADDL) induced dendritic spine loss in hippocampal neurons. Alzheimers Dement. 2019;15(7, Suppl): P1507.

43. Sevigny J, Chiao P, Bussière $T$, Weinreb $P H$, Williams $L$, Maier $M$, et al. The antibody aducanumab reduces $A \beta$ plaques in Alzheimer's disease. Nature. 2016:537(7618):50-6. https://doi.org/10.1038/nature19323.

44. Goldstein DM, Kuglstatter A, Lou Y, Soth MJ. Selective p38alpha inhibitors clinically evaluated for the treatment of chronic inflammatory disorders. J Med Chem. 2010;53(6):2345-53. https://doi.org/10.1021/jm9012906.

\section{Publisher's Note}

Springer Nature remains neutral with regard to jurisdictional claims in published maps and institutional affiliations.

Ready to submit your research? Choose BMC and benefit from:

- fast, convenient online submission

- thorough peer review by experienced researchers in your field

- rapid publication on acceptance

- support for research data, including large and complex data types

- gold Open Access which fosters wider collaboration and increased citations

- maximum visibility for your research: over $100 \mathrm{M}$ website views per year

At $\mathrm{BMC}$, research is always in progress.

Learn more biomedcentral.com/submissions 